\title{
Non-Linear Thickness Variation on the Thermally- Induced Vibration of a Rectangular Plate: A Spline Technique
}

\author{
Arun Kumar Gupta and Mamta \\ Department of Mathematics, M.S. College, Saharanpur 247001, UP, India
}

\begin{abstract}
The fourth order differential equation, governing the transverse motion of an elastic rectangular plate of a nonlinear thickness variation with a thermal gradient, has been analysed on the basis of classical plate theory employing the Quintic spline interpolation technique. An algorithm for computing the solution of this differential equation is presented for the case of equal intervals. The effect of the thermal gradient, together with taper constants on the natural frequencies of vibration, is illustrated for the first three modes of vibration for C-S-C-S and S-S-S-S rectangular plates.
\end{abstract}

\section{INTRODUCTION}

Plates of variable thickness having non-linear nature are of immense importance to engineers. For the design of aircraft wing sections, earthquake resistant structures, ship control devices, telephone receivers, radio telescopes, and other structures prone to vibration, a construction engineer needs the first few modes of vibration before finalizing a design.

With the advancement of technology, plates of varying thickness are being extensively used in civil, electronic, mechanical, aerospace, and marine engineering applications. It is necessary, therefore, to study the vibration behaviour of plates in order to avoid resonance excited by internal or external forces. Modern engineering structures are based on different types of design that involve various anisotropic and nonhomogeneous materials in the form of their structure components. Depending upon the requirement, durability, and reliability, materials are being developed so that they can provide better strength and efficiency. A number of researchers have worked on the free vibration analyses of plates of different shapes and varying thicknesses. Rectangular plates of non-linear varying thickness are widely used in different structures; however, they have been poorly studied, unlike linearlyvarying thickness. Rectangular plates of non-linear varying thickness with thermal gradients find various applications in the construction of modern high speed air craft. The vibration characteristics of such plates are of interest to the designers.

Various numerical techniques such as the Frobenius method, ${ }^{1}$ the finite-difference method, ${ }^{2}$ the simple polynomial approximation, ${ }^{3}$ the Galerkin method, ${ }^{4,5}$ the RayleighRitz method, ${ }^{6-8}$ characteristic orthogonal polynomials, ${ }^{9}$ the finite element method, ${ }^{10,11}$ and the Chebyshev collocation method, ${ }^{12,13}$ etc., have been employed to study the vibration characteristics of plates of various geometries. The above numerical methods, such as finite difference and finite element, require fine mesh size to obtain accurate results and are computationally expensive. The method of characteristic orthogonal polynomials and the Frobenius method require an appreciable number of terms for plates of varying thickness.

Here, a Quintic splines procedure is developed for obtaining the natural frequencies of rectangular plates of nonlinear varying thickness with the thermal gradient effect. The consideration of the present type of thickness variation was taken earlier by Gupta et al. for circular plates. ${ }^{14}$ The plate type structural components in aircraft and rockets have to operate under elevated temperatures, which cause non-homogeneity in the plate material; i.e., elastic constants of the material become functions of the space variables. In an up-to-date survey of the literature, these authors have come across various models to account for non-homogeneity in plate materials, as proposed by researchers dealing with vibration.

Akiyama and Kuroda discussed the fundamental frequencies of rectangular plates with linearly-varying thickness. ${ }^{15}$ Civalek discussed the fundamental frequency of isotropic and orthotropic rectangular plates with linearly-varying thickness by the discrete singular convolution method. ${ }^{16}$ Gupta et al. studied the thermal gradient effect on vibration of a nonhomogeneous orthotropic rectangular plate having bi-direction linear thickness variation. ${ }^{17}$ Gupta et al. did the vibration analysis of visco-elastic orthotropic parallelogram plate with linear thickness variation in both directions. ${ }^{18}$ Lal et al. studied the transverse vibrations of non-uniform orthotropic rectangular plates by the Quintic splines method. ${ }^{19}$ Gupta and Kaur studied the effect of the thermal gradient on the free vibration of clamped visco-elastic rectangular plates with linear thickness variation in both directions. ${ }^{20}$ Gupta and Khanna studied the vibration of visco-elastic rectangular plates with linear thickness variations in both directions. ${ }^{21}$

Gupta et al. observed the thermal effect on the vibration of a non-homogeneous orthotropic rectangular plate having bi-directional, parabolically-varying thickness. ${ }^{22}$ Tomar and Gupta studied the effects of thermal gradient on the frequencies of orthotropic rectangular plates of varying thickness in one and two directions. ${ }^{23,24}$ Gupta et al. studied the thermal effect on vibration of a parallelogram plate of linearlyvarying thickness and bi-directional linearly-varying thickness. ${ }^{25,26}$ Gupta et al. did the vibration study of a visco-elastic parallelogram plate of linearly-varying thickness. ${ }^{27}$ Raju studied the vibration of thin elastic plates of linearly-varying thickness. ${ }^{28}$ Grossi and Laura discussed the transverse vibrations of circular plates of linearly-varying thicknesses. ${ }^{29}$ Gupta and Ansari studied the effect of elastic foundation on the asym- 